\title{
DEVELOPMENT AND DIAGNOSTIC TOOLS IN THE FEEDBACK SYSTEM OF SRRC
}

\author{
C. H. Kuo, M.S. Yeh, W.K. Lau, K.H. Hu, Jenny Chen, K.T. Hsu \\ Synchrotron Radiation Research Center
}

No. 1 R\&D Road VI, Hsinchu Science-Based Industrial Park, Hsinchu 30077, Taiwan, R.O.C.

\begin{abstract}
Several DSP based feedback systems are developed in SRRC. These feedback systems include of longitudinal feedback system, global orbit feedback system and local orbit feedback system. Many development and diagnostic tools are necessary to be integrated in the feedback systems to support various beam study and system performance inspection. These systems are developed by the Commercial Off-The Shelf (COTS) extensive products those are to reduce development times, to improve integration quality, to reduce the cost of maintenance in various feedback systems. The implement features will be summarized in this report.
\end{abstract}

\section{INTRODUCTION}

The storage ring of SRRC is a $3^{\text {rd }}$ generation synchrotron light source, designed to store the beam current $200 \mathrm{~mA}$ at $1.5 \mathrm{GeV}$. Stable longitudinal dynamics are the most important performance issues of the storage ring. Longitudinal instabilities are prominent when the machine isn't optimized its operating conditions. However, to maintain optimized condition is not an easy job. Source of the longitudinal instabilities is mainly due to high order mode (HOM) of the RF cavities. Active longitudinal feedback system (LFB) was proposed to fight the instabilities. The system is in commissioning now.

Insertion devices play crucial roles in modern light sources. However, residue fields of insertion devices perturb orbit and lattice of storage ring is not negligible. Dynamic change parameters of insertion devices are essential to satisfy various user requirements. Any vibrations and orbit drift that lead to distortions in the closed orbit will result in a larger effective emittance. Together with the brightness reduction, unwanted beam motion that causes variation of the incident light position and angle. These effects can degrade the experimental advantages of synchrotron. Reduce various perturbations to an acceptable level by local bumps with orbit feedback system are main purpose of the orbit feedback system in the SRRC now.

The profile monitor is used in the SRRC for a long time. The old system hardware has no spare components. Data capture rate and analysis is slow. Upgrade to a friendly new system is necessary. This new system will support more stable and fast data acquisition for transverse beam profile analysis and beam diagnostic.

\section{LONGITUDE FEEDBACK SYSTEM}

The DSP technology advances drastically since first bunch-by-bunch feedback system deploys by PEPII/DAФNE/ALS collaboration [1]. New generation DSP chips provide 10-100 folds increase in computing horsepower than early DSPs. Number of DSPs can be reduced for new design. Experiences accumulated from these laboratories made us to design our system that is easier than before. Longitudinal feedback system was planed to combat with the longitudinal instabilities [2, 3] for the storage ring of SRRC. Plan to replace two existing DORIS cavities by a single cell Cornell superconductivity RF (SRF) cavity at the storage ring is underway. Nearly HOM free properties are promising of SRF cavity. This project is also accompanied with 400 $\mathrm{mA}$ upgrade goals. Longitudinal feedback system is used to damp residues longitudinal instabilities after SRF installation is still necessary. Powerful diagnostics of LFS are useful to study the beam properties under conventional RF as well as SRF environments.

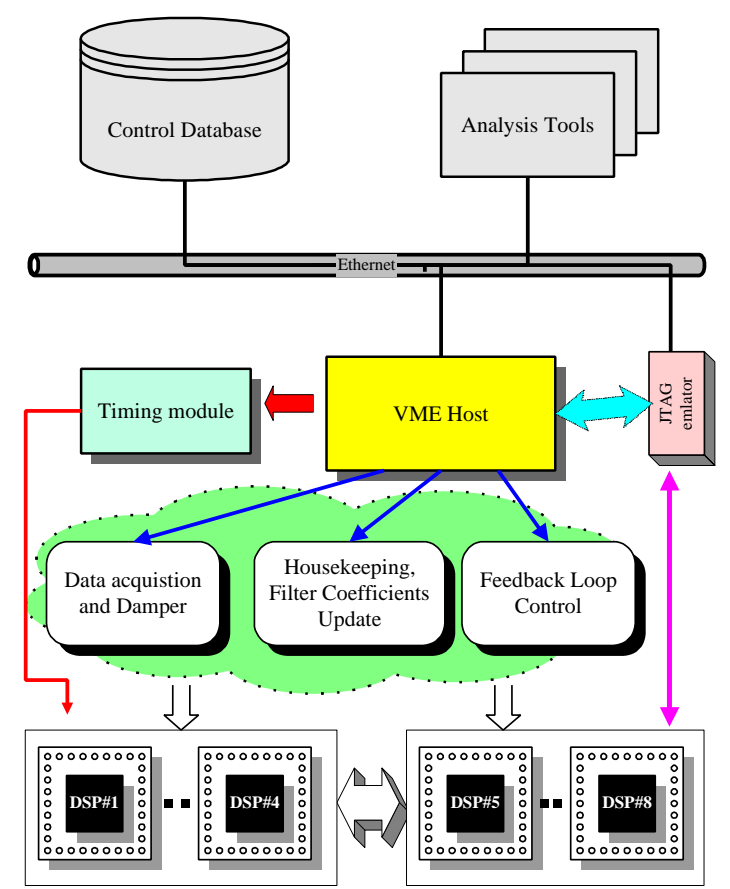

Figure 1: Software task.

\subsection{Software Task}

The software structure of longitudinal multi-bunch feedback system (LFB) consists of a digital signal 
processor array to execute feedback algorithm and to capture raw data for diagnostic purposes. In such environment, major software components includes, (1) feedback control algorithm, filtering and house keeping on DSP, (2) DSP developing environment is on PC, (3) diagnostics toolkits is on PC and VME host, (4) user interface on PC and workstation. The system block is shown in figure 1.

\subsection{Timing Arrangement of Feedback Software}

The basic cycle time of the feedback software is decided by sampling rate of the system. Sampling frequency is equal to revolution frequency of the storage ring be divided by decimation factor. During every sampling period, the ADC unit shall send phase oscillation data to DSPs FIFO by COMM port. The DSPs access phase error signals from FIFO when synchronization signal is active. The data is sent to output FIFO after applied control rule. The remained time is for housekeeping purpose. The DAC convert corrected signal and send to back end electronics and power amplifier. The timing arrangement is shown in figure 2.

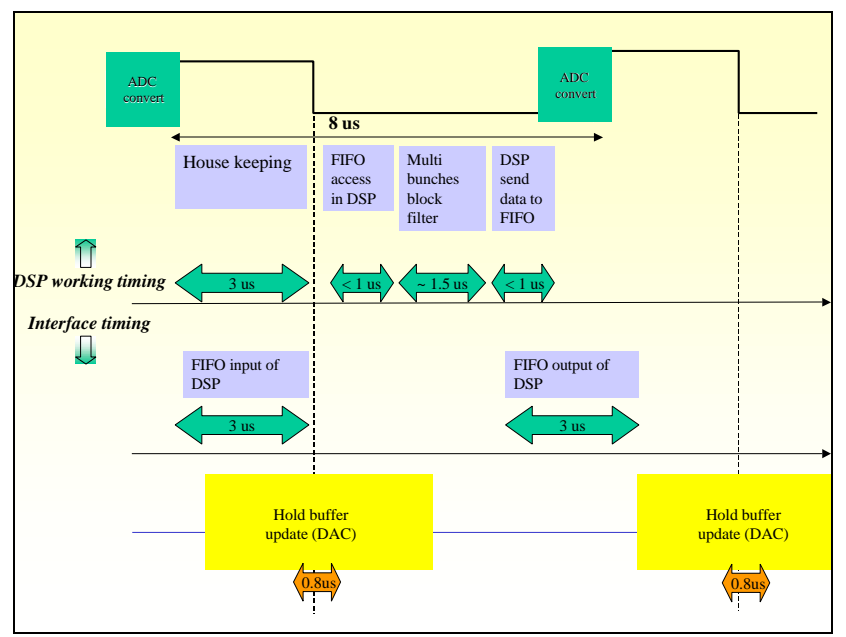

Figure 2: Timing arrangement of software components.

\subsection{Operation}

The feedback software includes grow/damp and debugger mode for present implementation. The grow/damp mode is used to extract damping and growth time of various beam modes. This mode can reverse its operation sequence as damp/grow mode by filter coefficient swap. The debugger mode is supported by hardware diagnostic request. Data can be updated automatically in this mode.

The operation of hold off can postpone data acquisition time. The operation of pre-access samples can update coefficients of aux after samples of setting. The hold off and pre-access samples are only effective in the grow/damp mode.

The reset button is to initialization the operation of $\mathrm{ADC} / \mathrm{DAC}$ and DSP. The batch data capture is support to all bunches information access by network. These data will be saved in a file and analyzed by Prabhakar's MATLAB code $[4,5]$. The operation graphical interface can be operated in WindowNT/98 and UNIX workstation as shown in figure 3 .

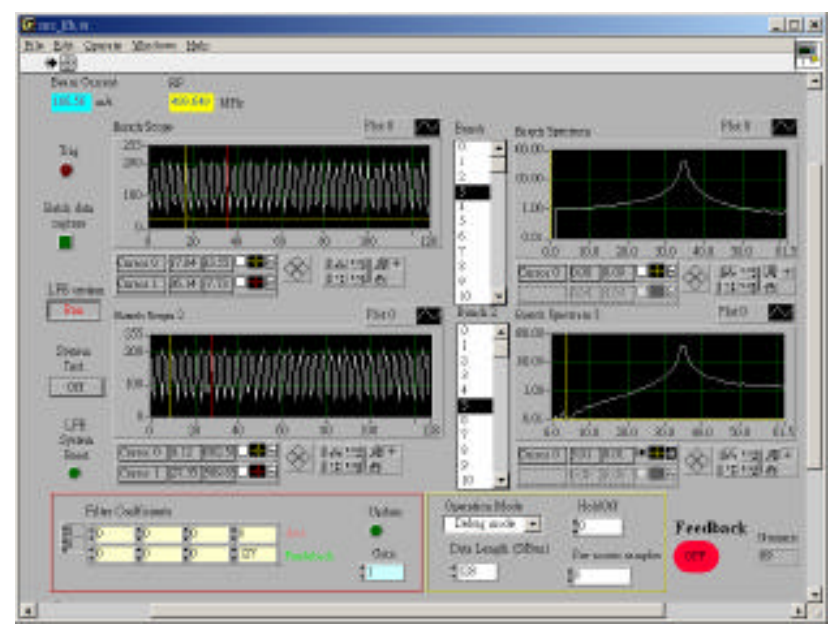

Figure 3: Graphical user interface of LFB system operation.

\section{ORBIT FEEDBACK SYSTEM}

The orbit feedback system operated routinely since 1996. The original tools are out-of-date due to the fast evolution of technology. It is difficult to maintain. Old system isn't also satisfying demanding applications. The development environment is gradually ported to new platform. The graphic user interface of response matrix measurement is ported at first, as shown in the figure 4.

New version software is developed in Pentium platform running Linux operating system. It also supports orbit information display, control algorithm development and integration with commercial products for the applications of storage ring in future.

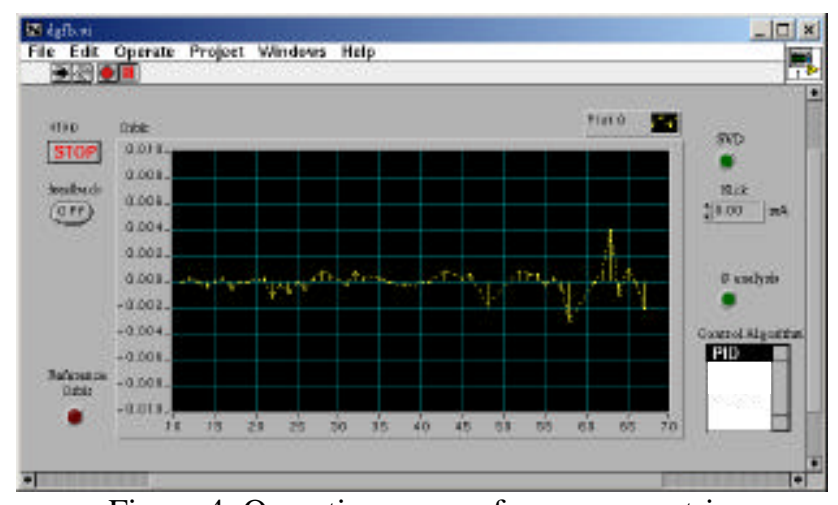

Figure 4: Operation menu of response matrix measurement.

\section{PROFILE MONITOR SYSTEM}

The profile monitor is applied to capture and analysis of synchrotron radiation profile for machine operation. 
The original system is working with the Intel 486 running MS-DOS system. The frame grabber is an ISA bus interface card. The update rate is about 2 frames per second. The new system is a Pentium based PC running Windows NT. The frame grabber was upgraded to PCI bus interface card. It supports multiple console connection via control network. The update rate achieves 15 frames per second. The transaction consists of network access, frame capture and analysis. The beam parameters are also shown in the same page. Since, the synchrotron radiation monitor is located at dispersion region; energy oscillation will cause horizontal beam size fluctuation randomly. This tool is import to monitor the stability of stored beam.

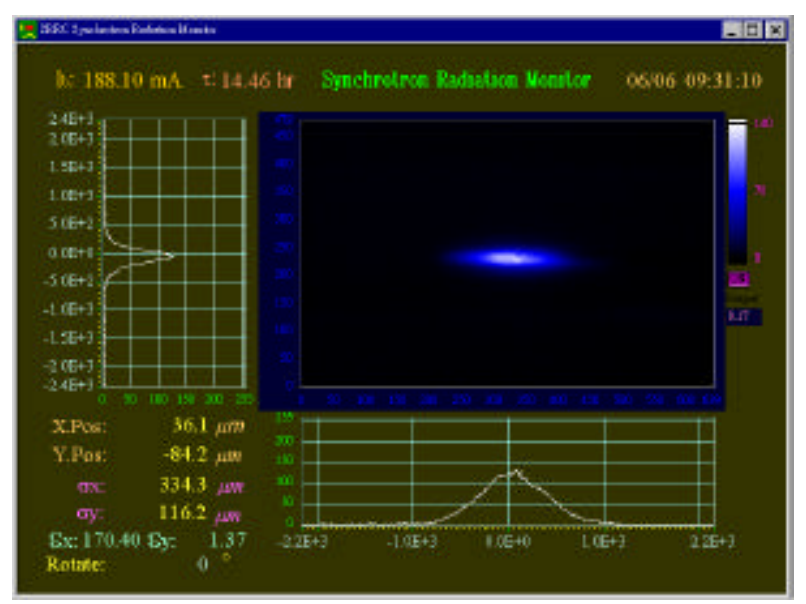

Figure 5: Graphic user interface of profile monitor.

\section{CONCLUSION}

The longitudinal instabilities at the storage ring of SRRC are a dominated factor, which deteriorate the beam quality. Various measures were adopted to mitigate the effect of these instabilities. From the operational experience from six LFB installations, which is the fruitful results of SLAC/DA $\Phi$ NE/ALS collaboration reveal that LFB system is an effective tool to fight longitudinal instabilities. The LFB system of SRRC is expected deploy soon. Most of the difficulties were solved recently. Commissioning is in progress now. The system is expected helpful to cure longitudinal instabilities at the storage ring of SRRC. Even after HOM free superconductivity RF cavity is installed, the LFB system is still useful to damp residue longitudinal instabilities. Powerful of transient domain diagnostic are very useful to investigate various instabilities issues in pre and post SRF era. Adopt alias sampling techniques made the LFB system possible as a tools for the transient domain transverse instabilities observation, it is promising for the studies of transverse instabilities and ion effect.

Orbit feedback system upgrade is on-going. Future system will include of orbit server crate, power supply setting crate, DSP crate and analysis tools. The DSP crate is shutdown as if feedback is only turned off and no more bad effect for orbit. This configuration is defined that the new system will be more advanced system security than before. The response matrix of DSP will be dynamically updated by console. This environment will be easy to connect with on-line analysis tools and modelling, such as MATLAB and LABVIEW.

\section{REFERENCES}

[1] J. D. Fox, "Programmable DSP-Based multi-bunch feedback - Operational Experience from Six Installations", AIP Conference Proc. Vol. 546, p.226 2000.

[2] W. K. Lau, S. J. Lin, M. S. Yeh, L. H. Chang, T. T. Yang, K. T. Hsu, M. H. Wang, C. C. Kuo: Development Of A Digital Longitudinal Damper For The TLS Storage Ring, Proceedings of 1997 IEEE Particle Accelerator Conference, Vancouver, 1997.

[3] W. K. Lau, L. H. Chang, K. T. Hsu, C. H. Kuo, J. A. Li, M. S. J. Lin, T. T. Yang. M. S. Yeh: Progress of the TLS Longitudinal Feedback System and Associated Beam Longitudinal-Dynamics Studies, Proceedings of 1999 IEEE Particle Accelerator Conference, New York, 1999.

[4] S. Prabhakar, "New Diagnostics and Cures for Coupled-Bunch Instabilities", SLAC-Report-554, February 2000.

[5] S. Prabhakar, J. D. Fox, D. Teytelman, and SA. Young, "Phase space tracking of coupled-bunch instabilities", Phy. Rev. Special Topics - Accel. and Beams, PRST-AB 2, 084401 (1999) and reference therin. 